\title{
Online monitoring of chlorophyll a fluorescence in Nannochloropsis oceanica to assess photochemical changes and the onset of lipid accumulation during nitrogen deprivation
}

\author{
Mariana Carneiro ${ }^{1}$, Graziella Chini Zittelli ${ }^{2}$, Bernardo Cicchi $^{3}$, Elefterios Touloupakis ${ }^{4}$, \\ Cecilia Faraloni $^{3}$, Inês Maia ${ }^{5}$, Hugo Pereira ${ }^{6}$, Tamára Santos ${ }^{5}$, Francisco Xavier Malcata ${ }^{1}$, \\ Ana Otero ${ }^{7}$, João Varela ${ }^{5}$, and Giuseppe Torzillo ${ }^{8}$ \\ ${ }^{1} \mathrm{LEPABE}$ \\ ${ }^{2}$ National Research Council \\ ${ }^{3}$ National Research Council Department of Biological Agricultural and Food Sciences \\ ${ }^{4}$ National Research Council Department of Earth System Science and Environment \\ Technologies \\ ${ }^{5}$ CCMAR \\ ${ }^{6} \mathrm{GreenCoLab}$ \\ ${ }^{7}$ Universidade de Santiago de Compostela \\ ${ }^{8}$ Centro de Investigación en Ciencias del Mar y Limnologia
}

May 21, 2021

\begin{abstract}
In this work, we applied online chlorophyll a fluorescence measurements to monitor the changes in the photochemical parameters both in nitrate-deplete and nitrate-replete cultures of Nannochloropsis oceanica, in addition to biochemical parameters such as growth, lipid, fatty acid, and pigment contents. Under nitrate-replete conditions, growth was promoted along with pigment content, while total lipid content and fatty acid saturation level diminished. Under nitrate-deplete conditions, cultures showed an increased de-epoxidation state of the xanthophyll cycle pigments. Fast transients revealed a poor processing efficiency for electron transfer beyond QA, which was in line with the low electron transport rate due to nitrate depletion. Lipid content and the de-epoxidation state were the first biochemical parameters triggered by the change in nutrient status, which coincided with a $20 \%$ drop in the online effective quantum yield of PSII ( $\Delta \mathrm{F} / \mathrm{Fm}$ '), and a raise in the $\mathrm{Vj}$ measurements. A good correlation was found between the changes in $\Delta \mathrm{F} / \mathrm{Fm}$ ' and lipid content $(\mathrm{r}=-0.96, \mathrm{p}<0.01)$. The results confirm the reliability and applicability of online fluorescence measurements to monitor lipid induction in N. oceanica.
\end{abstract}

\section{Hosted file}

MS_FINAL(mod).docx available at https://authorea.com/users/415057/articles/522972online-monitoring-of-chlorophyll-a-fluorescence-in-nannochloropsis-oceanica-to-assessphotochemical-changes-and-the-onset-of-lipid-accumulation-during-nitrogen-deprivation 

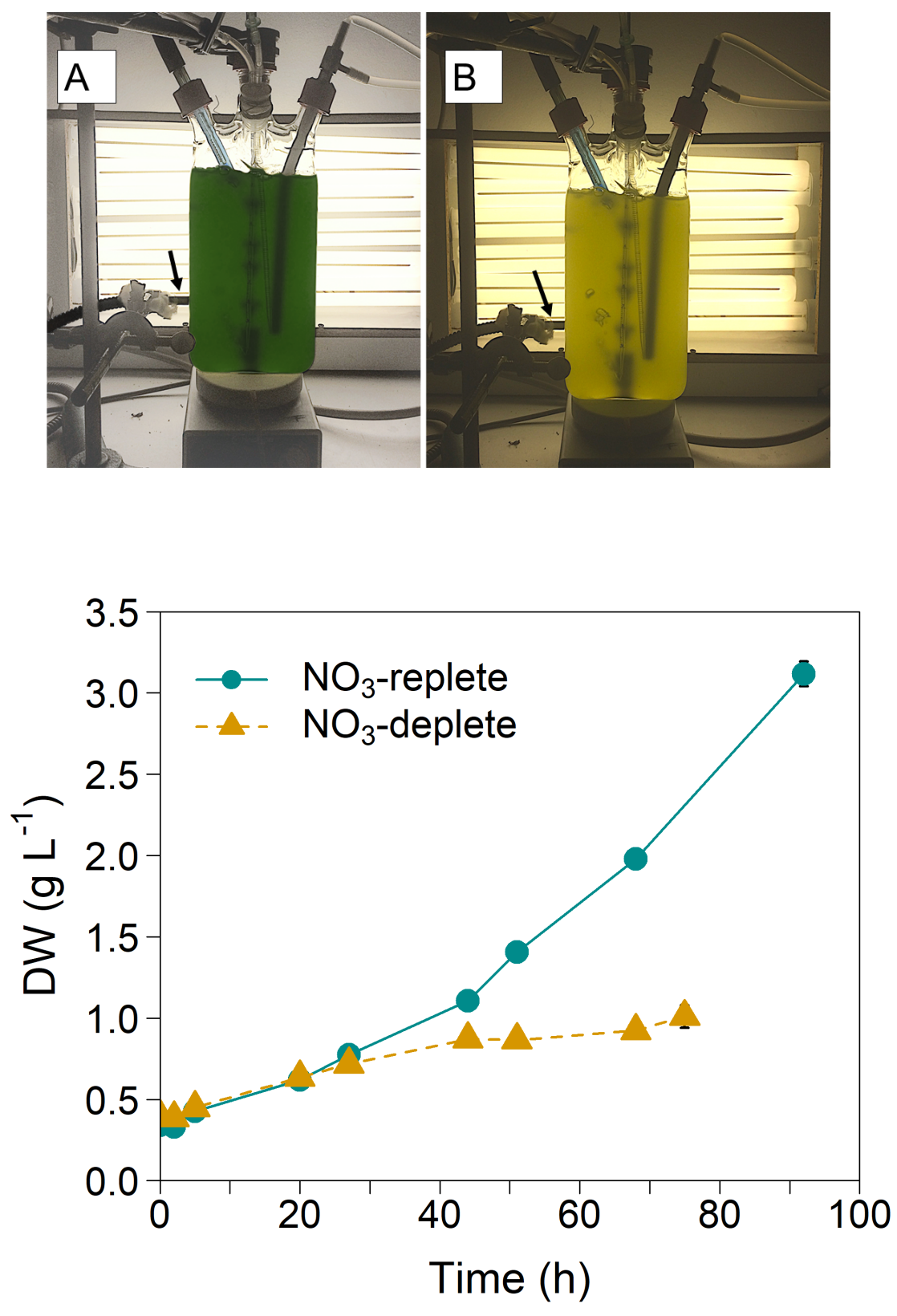

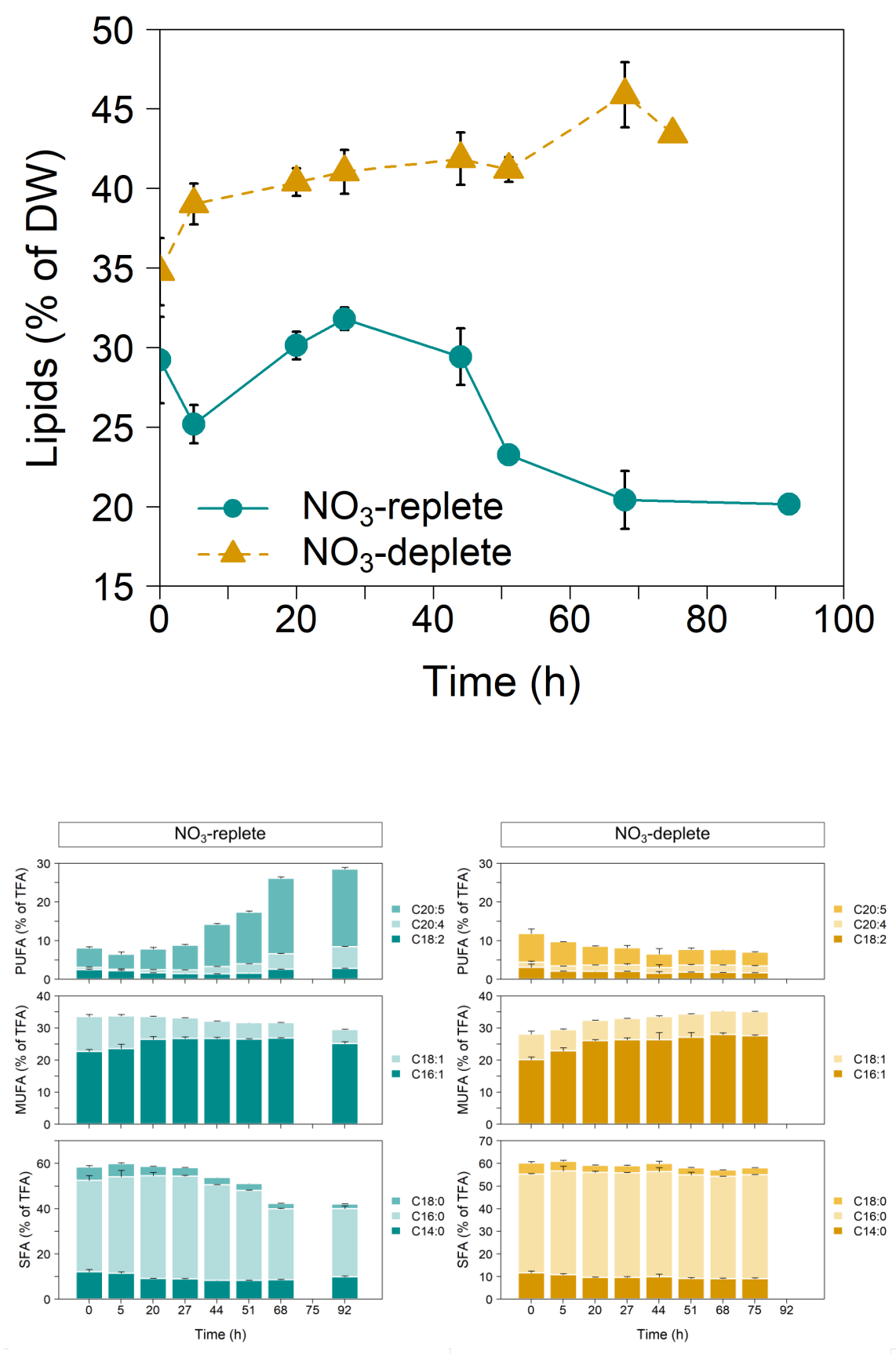

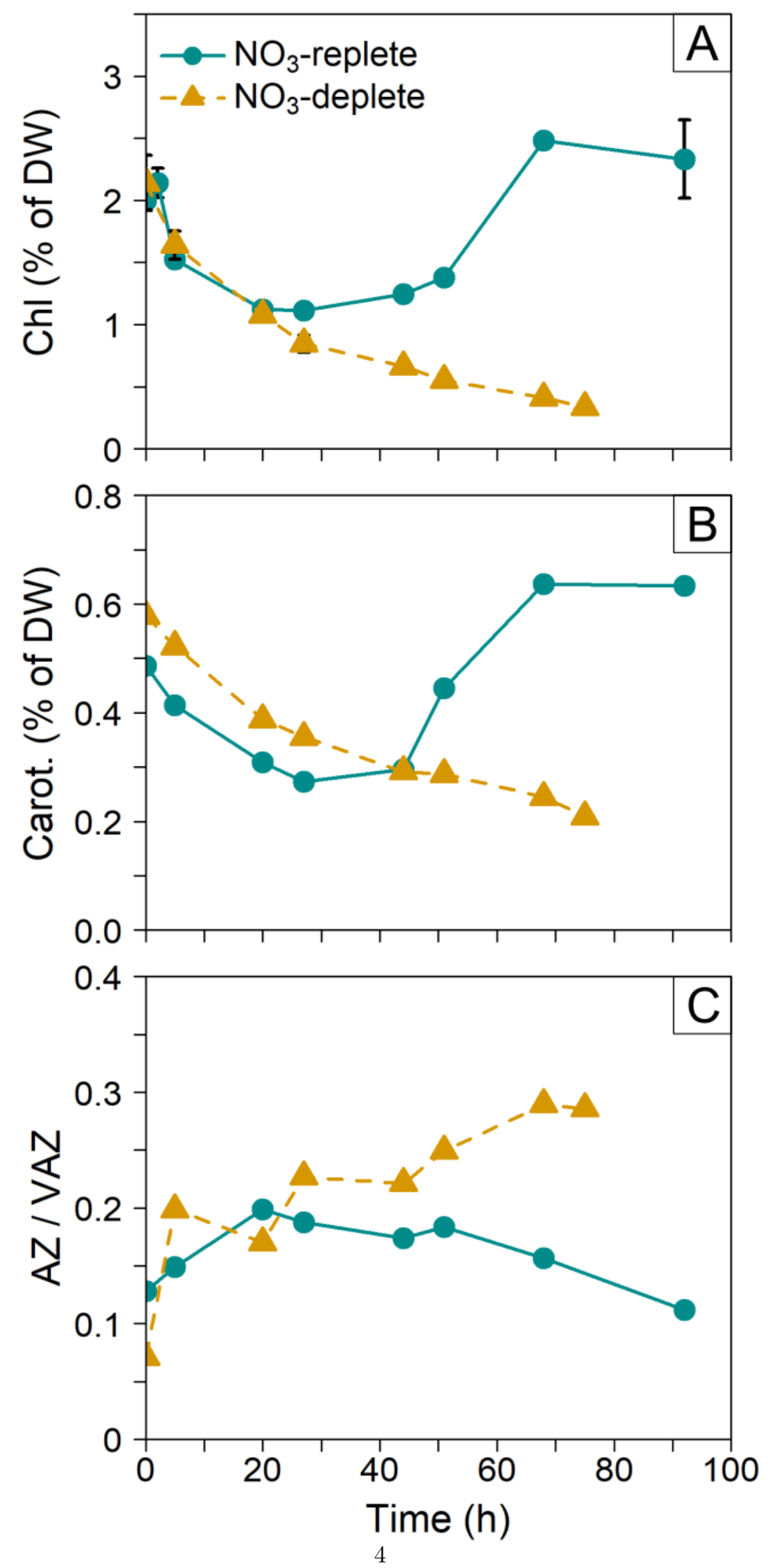

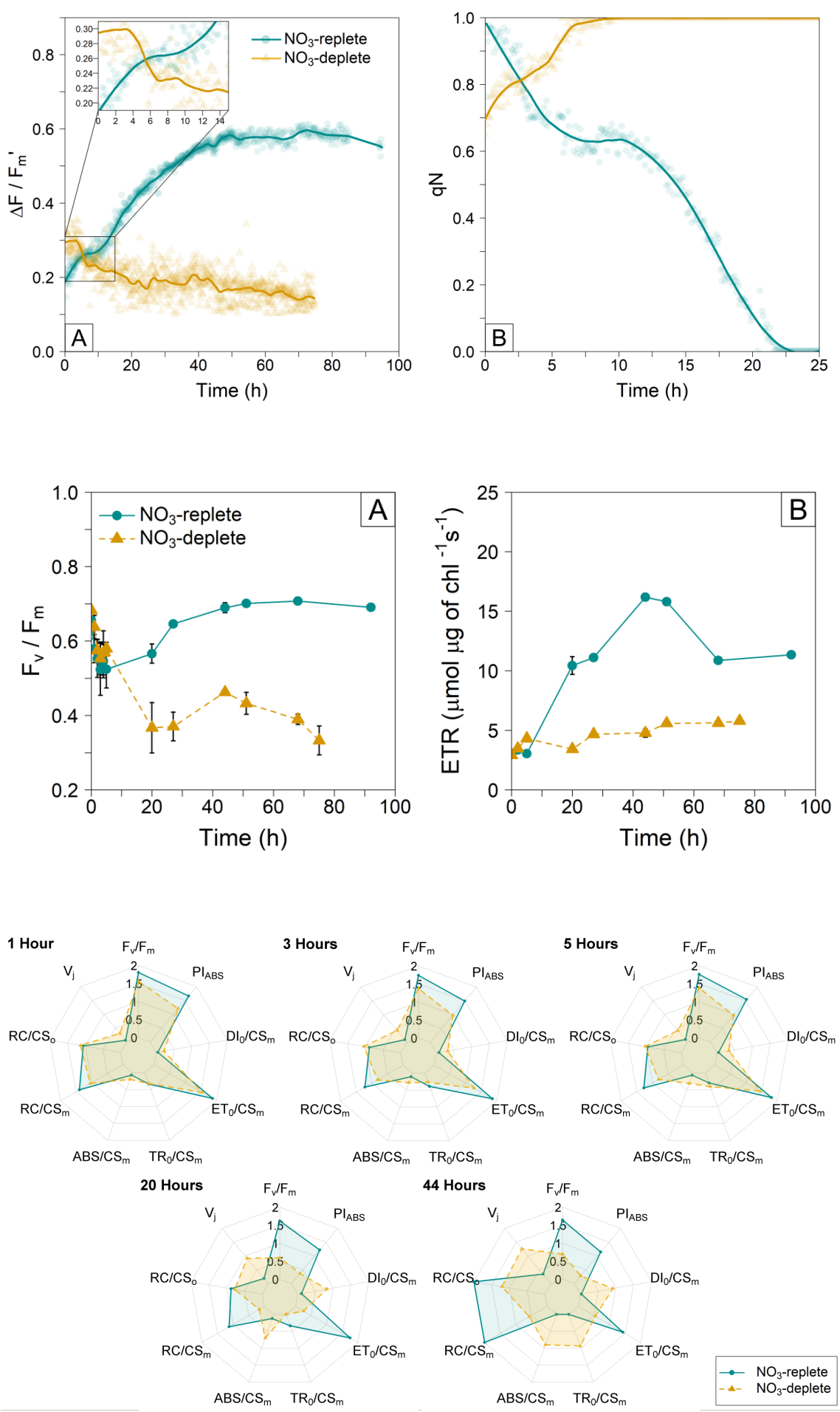

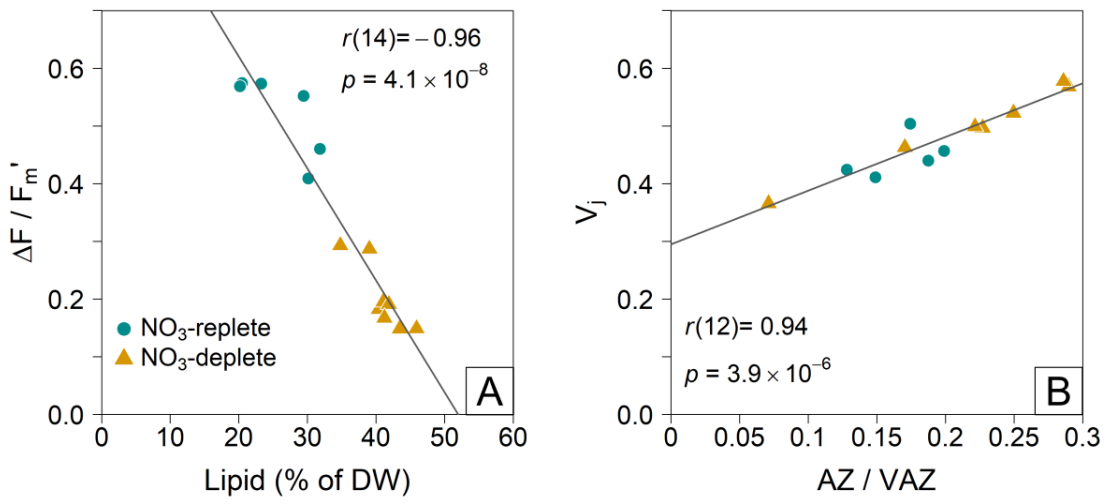